\title{
On State Estimation for Discrete Time-Delayed Memristive Neural Networks under the WTOD Protocol: A Resilient Set-Membership Approach
}

\author{
Hongjian Liu, Zidong Wang, Fellow, IEEE, Weiyin Fei and Hongli Dong
}

\begin{abstract}
In this paper, a resilient set-membership approach is put forward to deal with the state estimation problem for a sort of discrete-time memristive neural networks (DMNNs) with hybrid time-delays under the Weighted Try-Once-Discard protocol (WTODP). The WTODP is utilized to mitigate unnecessary network congestion occurring in the channel between the DMNNs and the state estimator. In order to ensure resilience against possible realization errors, the estimator gain is permitted to undergo some norm-bounded parameter drifts. Our objective is to design a resilient set-membership estimator (RSME) that is capable of resisting gain variations and unknown-but-bounded noises by confining the estimation error to certain ellipsoidal regions. By resorting to the recursive matrix inequality technique, sufficient conditions are acquired for the existence of the expected RSME and, subsequently, an optimization problem is formalized by minimizing the constraint ellipsoid (with respect to the estimation error) under the WTODP. At last, numerical simulation is carried out to validate the usefulness of the RSME.
\end{abstract}

Index Terms-Discrete-time memristive neural networks, setmembership state estimation, hybrid time-delays, resilient state estimation, weighted try-once-discard protocol.

\section{INTRODUCTION}

The past decade has seen a recurring research interest in memristive neural networks (MNNs) because of their extensive applications in various subjects, e.g. recognition of patterns [33], processing of digital images [11], and brain

This work was supported in part by the National Natural Science Foundation of China under Grants 61873058, 61873148 and 61933007, the AHPU Youth Top-notch Talent Support Program of China under Grant 2018BJRC009, the Natural Science Foundation of Universities in Anhui Province of China under Grant gxyqZD2019053, the Heilongjiang Postdoctoral Sustentation Fund of China under Grant LBH-Z19048, the Royal Society of the UK, and the Alexander von Humboldt Foundation of Germany. (Corresponding author: Zidong Wang.)

H. Liu is with the Key Laboratory of Advanced Perception and Intelligent Control of High-end Equipment, Ministry of Education, Anhui Polytechnic University, Wuhu 241000, China, and is also with the Artificial Intelligence Energy Research Institute, Northeast Petroleum University, Daqing 163318, China. (Email: hjliu1980@gmail.com)

Z. Wang is with the College of Electrical Engineering and Automation, Shandong University of Science and Technology, Qingdao 266590, China, and is also with the Department of Computer Science, Brunel University London, Uxbridge, Middlesex, UB8 3PH, United Kingdom. (Email: Zidong. Wang@brunel.ac.uk)

W. Fei is with the Key Laboratory of Advanced Perception and Intelligent Control of High-end Equipment, Ministry of Education, Anhui Polytechnic University, Wuhu 241000, China, and is also with the School of Mathematics and Physics, Anhui Polytechnic University, Wuhu 241000, China. (Email: wy fei@ahpu.edu.cn)

H. Dong is with the Artificial Intelligence Energy Research Institute, Northeast Petroleum University, and is also with the Heilongjiang Provincial Key Laboratory of Networking and Intelligent Control, Northeast Petroleum University, Daqing 163318, China. (Email: shiningdhl@vip.126.com) emulation [26]. The key feature of MNNs is their strong dependence of the connection weights (CWs) on the neuron states, which leads to complicated dynamic behaviors of MNNs as compared to the conventional recurrent neural networks (RNNs). Accordingly, the analysis problems (e.g. stability, convergence and synchronization) of many kinds of dynamics of MNNs have aroused much interest, and some pioneering work has been available in [37], [45]. Almost all existing results, however, have been concerned with the continuoustime MNNs despite the nowadays popularity of the digitization of information sequences in engineering practice, and this gives rise to the necessity of utilizing discrete-time RNNs when handling sequence-based missions. Up to now, little attention has been drawn onto the DMNNs simply because of essential difficulties in dealing with switching behaviors that are inherently state-dependent in the discrete-time setting.

State estimation is a fundamental yet crucial research issue in engineering applications on neural networks (NNs) [16], [27], [29], [40], [43], [44]. In fact, for many NN applications, it is indispensable for the network states to be utilized to fulfil the requirements for monitoring, approximation and optimization [4], [8], [32]. Unfortunately, due mainly to resource constraints and technical limits, the network states are usually unavailable to the end user but only the measurement outputs can be accessible. In addition, when analyzing DMNN-related dynamics, the frequently adopted analysis techniques (e.g. the nonsmooth analysis method and differential inclusions theory) for continuous-time MNNs become futile, and this leads to an urgent demand for developing new techniques appropriate for DMNNs. As such, much research enthusiasm has recently been attracted towards the state estimation problems for DMNNs suffering from hybrid (i.e. both discrete and distributed) time-delays with some inspiring results published in the recent literature [18].

In practice, MNNs can be realized via very large scale integration circuits (VLSICs) where the CWs are executed via memristors [37]. Basically speaking, VLSICs are easily susceptible to both device noises (e.g. the flicker, shot and thermal noises) and ambient noises (e.g. the crosstalk, power and/or ground bounce, and substrate noises). Particularly, the ambient noises, if not adequately addressed, could significantly degrade the VLSIC performance and reliability [31]. From a practical point of view, we assume the ambient noises to be deterministic, unknown-but-bounded within certain ellipsoidal regions (CERs). In this case, the set-membership (ellipsoidal) filtering method becomes especially suitable to constrain sys- 
tem states in CERs so as to assure satisfactory state estimation performance [17]. Although a vast body of set-membership state estimation work has been presented (see e.g. [20], [49]), relevant results on MNNs have been scattered, let alone the discrete-time setting is also involved.

It should be pointed out that, even though some attempts have been initiated on the state estimation issues for MNNs (see e.g. [18], [19]), little attention has been paid to the issue of limited communication under resource constraints between the estimator and the network output [3]. Because of the large size of MNNs and the high-degree complexity of the to-do tasks, the volume of the network output data could become considerably high, which has posed great challenges (e.g. data collisions and communication congestion) onto the transmission networks of limited capacity. To handle the network-induced challenges, an effective measure that has been favorably taken by industry is to leverage the communication scheduling protocols in order to regulate the network traffic, see [5], [28], [34], [50], [51]. Among various protocols that have been in use so far, the Weighted Try-Once-Discard protocol (WTODP) has proven to be a particularly attractive scheduling strategy in allocating network resources because of its dynamic scheduling behavior based on the significance of different missions [30]. As such, it is of practical significance to explore how the WTODP can be leveraged to coordinate the considerable data transmission between the MNN and the estimator, and this constitutes one of our motivations in the present investigation.

The biological or circuit implementation of NNs is often confronted by various types of time-delays for the reasons of finite signal transmission and switching speeds in biological systems [1], [12], [15], [39], [47]. It has been acknowledged that time-delays can cause undesirable system performance including instability and oscillation, and thus plenty of research effort has been spent on analyzing the dynamical behaviors of NNs with various time-delays (constant, timevarying, discrete, distributed, mixed delays) where the majority build themselves on existing techniques such as the descriptor model transformation [6], integral inequality [14], etc. For the stability issue of delayed continuous-time MNNs, fruitful results have been published by combining the proceeding techniques and the set-valued mapping theory, see [37]. However, when it comes to the delayed DMNNs, corresponding results have been rarely reported due typically to the mathematical challenges embedded in disposing of the coupling among the state-dependent parameters, the time-delays, the scheduling protocol, and the estimator. Accordingly, one of the primary motivations here is to examine the set-membership state estimation problem (SEP) for delayed DMNNs under the WTODP.

When realizing filter/controller algorithms in traditional system design, an implicit assumption is that filter/controller gains are exactly implemented. This assumption, unfortunately, goes against the engineering practice as the actual execution of the filter/controller gains might undergo parameter variations induced by many factors including the analoguedigital conversion, round-off errors, and finite resolution of instruments [21]. Such kind of gain variations/drifts, though possibly small, would undoubtedly impair the corresponding controller/filter performance [13], [38]. As such, a growing body of work has been reported on the design of the resilient state estimation algorithms that are of insensitivity to the estimator gain variations [35], [41], [42], [46]. Nevertheless, to our best knowledge, very few results have been acquired so far on the resilient SEPs for DMNNs, not to mention the case where the hybrid time-delays (HTDs) (consisting both discrete and distributed time-delays), unknown-but-bounded noises (UBBNs) and WTODP are all involved.

In this paper, we aim at developing a resilient setmembership estimator (RSME) for DMNNs with HTDs under the WTODP. The primary contributions we make in this paper are outlined as threefold. 1) A RSME is developed to mirror the alleviation of the adverse impact induced by the random gain variation in the actual estimator implementation. 2) A unified estimation scheme is built to cope with the mathematical complexities resulting from the state-dependent switching behaviors, HTDs, WTODP and gain variations. 3) In the designed filtering framework, the filter gain is obtained with the help of a bank of recursive matrix inequalities (RMIs) whose solutions are provided by standard software packages.

The rest of this article is highlighted as follows. Section II formulates the concerned filtering problem. Section III presents the design procedure of the filter whose existence is guaranteed by the derivation of some sufficient conditions. An example is given in Section IV to show the feasibility of our RSME, and some remarks are concluded in Section V.

Notation $\mathbb{R}^{n}$ and $\mathbb{R}^{n \times m}$ define, respectively, the $n$ dimensional Euclidean space and the set of all $n \times m$ real matrices. $\mathbb{N}^{+}$denotes the set of all nonnegative integers. $I$ denotes identity matrices with compatible dimensions. $A>B$ (or $A \geq B$ ) implies that $A-B$ is positive-definite (or semipositive-definite). $A^{T}$ represents the transpose of $A$. $\operatorname{diag}\{\cdots\}$ is a block-diagonal matrix. $\delta(\cdot) \in\{0,1\}$ is the Dirac delta function.

\section{Problem Formulation}

\section{A. Memristive Neural Network Model}

Consider a typical DMNN with HTDs:

$$
\left\{\begin{aligned}
z(t+1)= & D(z(t)) z(t)+A(z(t)) f(z(t)) \\
& +B(z(t)) g\left(z\left(t-\tau_{1}\right)\right) \\
& +C(z(t)) \sum_{\iota=1}^{\tau_{2}} \mu_{\iota} h(z(t-\iota))+L_{1} v(t), \\
y(t)= & M z(t)+L_{2} v(t), \\
z(\varsigma)= & \phi_{0}(\varsigma), \quad \varsigma=-\tau,-\tau+1, \cdots,-1,0
\end{aligned}\right.
$$

where

$$
\begin{aligned}
z(t) & =\left[\begin{array}{llll}
z_{1}(t) & z_{2}(t) & \cdots & z_{n}(t)
\end{array}\right]^{T} \\
y(t) & =\left[\begin{array}{llll}
y_{1}(t) & y_{2}(t) & \cdots & y_{m}(t)
\end{array}\right]^{T} \\
v(t) & =\left[\begin{array}{llll}
v_{1}(t) & v_{2}(t) & \cdots & v_{q}(t)
\end{array}\right]^{T} \\
D(z(t)) & =\operatorname{diag}\left\{d_{1}\left(z_{1}(t)\right), d_{2}\left(z_{2}(t)\right), \cdots, d_{n}\left(z_{n}(t)\right)\right\}
\end{aligned}
$$


are the neuron state vector, the measurement output vector, the exogenous disturbance, and the self-feedback matrix, respectively; $A(z(t))=\left(a_{i j}\left(z_{i}(t)\right)\right)_{n \times n}, B(z(t))=\left(b_{i j}\left(z_{i}(t)\right)\right)_{n \times n}$ and $C(z(t))=\left(c_{i j}\left(z_{i}(t)\right)\right)_{n \times n}$ are CWs; and

$$
\begin{aligned}
& f(z(t)) \triangleq\left[\begin{array}{llll}
f_{1}\left(z_{1}(t)\right) & f_{2}\left(z_{2}(t)\right) & \cdots & f_{n}\left(z_{n}(t)\right)
\end{array}\right]^{T}, \\
& g(z(t)) \triangleq\left[\begin{array}{llll}
g_{1}\left(z_{1}(t)\right) & g_{2}\left(z_{2}(t)\right) & \cdots & g_{n}\left(z_{n}(t)\right)
\end{array}\right]^{T}, \\
& h(z(t)) \triangleq\left[\begin{array}{llll}
h_{1}\left(z_{1}(t)\right) & h_{2}\left(z_{2}(t)\right) & \cdots & h_{n}\left(z_{n}(t)\right)
\end{array}\right]^{T}
\end{aligned}
$$

are the nonlinear neuron activation functions (AFs); $\tau_{1}$ and $\tau_{2}$ are the constant discrete and distributed time-delays, respectively; $\phi_{0}(\varsigma)(\varsigma=-\tau,-\tau+1, \cdots,-1,0)$ are initial conditions with $\tau \triangleq \max \left\{\tau_{1}, \tau_{2}\right\} ; L_{1}, L_{2}$ and $M$ are known matrices of compatible dimensions.

Similar to [18], the state-dependent functions $d_{i}\left(z_{i}(t)\right)$, $a_{i j}\left(z_{i}(t)\right), b_{i j}\left(z_{i}(t)\right)$ and $c_{i j}\left(z_{i}(t)\right)$ are

$$
\begin{gathered}
d_{i}\left(z_{i}(\cdot)\right)= \begin{cases}\hat{d}_{i}, & \left|z_{i}(\cdot)\right|>\ell_{i}, \\
\check{d}_{i}, & \left|z_{i}(\cdot)\right| \leq \ell_{i},\end{cases} \\
\kappa_{i j}\left(z_{i}(\cdot)\right)= \begin{cases}\hat{\kappa}_{i j}, & \left|z_{i}(\cdot)\right|>\ell_{i}, \\
\check{\kappa}_{i j}, & \left|z_{i}(\cdot)\right| \leq \ell_{i},\end{cases}
\end{gathered}
$$

where $\kappa \in\{a, b, c\}$, the switching jumps satisfy $\ell_{i}>0,\left|\hat{d}_{i}\right|<$ $1,\left|\check{d}_{i}\right|<1$, and $\hat{\kappa}_{i j}$ and $\check{\kappa}_{i j}$ are known constants.

Based on (2), we first define following switching functions (SFs):

$$
\begin{aligned}
& \vartheta_{i i}^{d}\left(z_{i}(\cdot)\right) \triangleq\left\{\begin{array}{rr}
1, & \left|z_{i}(\cdot)\right|>\ell_{i}, \\
-1, & \left|z_{i}(\cdot)\right| \leq \ell_{i},
\end{array}\right. \\
& \vartheta_{i j}^{\kappa}\left(z_{i}(\cdot)\right) \triangleq\left\{\begin{array}{rr}
1, & \left|z_{i}(\cdot)\right|>\ell_{i}, \\
-1, & \left|z_{i}(\cdot)\right| \leq \ell_{i},
\end{array}\right.
\end{aligned}
$$

and $\vartheta_{i j}^{d}\left(z_{i}(t)\right) \triangleq 0$ for $i \neq j$.

Denote

$$
\begin{aligned}
\underline{d}_{i} & \triangleq \min \left\{\hat{d}_{i}, \check{d}_{i}\right\}, \\
\bar{d}_{i} & \triangleq \max \left\{\hat{d}_{i}, \check{d}_{i}\right\}, \\
\underline{\kappa}_{i j} & \triangleq \min \left\{\hat{\kappa}_{i j}, \check{\kappa}_{i j}\right\}, \\
\bar{\kappa}_{i j} & \triangleq \max \left\{\hat{\kappa}_{i j}, \check{\kappa}_{i j}\right\} .
\end{aligned}
$$

In addition, we introduce the following matrices:

$$
\begin{aligned}
\underline{D} & \triangleq \operatorname{diag}\left\{\underline{d}_{1}, \underline{d}_{2}, \cdots, \underline{d}_{n}\right\}, \\
\bar{D} & \triangleq \operatorname{diag}\left\{\bar{d}_{1}, \bar{d}_{2}, \cdots, \bar{d}_{n}\right\}, \\
D_{0} & \triangleq(\underline{D}+\bar{D}) / 2, \\
\underline{S} & \triangleq\left(\underline{\kappa}_{i j}\right)_{n \times n}, \\
\bar{S} & \triangleq\left(\bar{\kappa}_{i j}\right)_{n \times n}, \\
S_{0} & \triangleq(\underline{S}+\bar{S}) / 2
\end{aligned}
$$

where $S \in\{A, B, C\}$. Thus, $D(z(t)), A(z(t)), B(z(t))$ and $C(z(t))$ are rewritten as

$$
\begin{aligned}
& D(z(t))=D_{0}+\Delta D(t), \quad A(z(t))=A_{0}+\Delta A(t), \\
& B(z(t))=B_{0}+\Delta B(t), \quad C(z(t))=C_{0}+\Delta C(t)
\end{aligned}
$$

with

$$
\Delta D(z(t)) \triangleq \mathcal{H}_{d} \vartheta^{d}(z(t)) E_{d}
$$

$$
\begin{aligned}
& \Delta A(z(t)) \triangleq \mathcal{H}_{a} \vartheta^{a}(z(t)) E_{a}, \\
& \Delta B(z(t)) \triangleq \mathcal{H}_{b} \vartheta^{b}(z(t)) E_{b}, \\
& \Delta C(z(t)) \triangleq \mathcal{H}_{c} \vartheta^{c}(z(t)) E_{c}
\end{aligned}
$$

where

$$
\begin{aligned}
& \mathcal{H}_{r} \triangleq\left[\begin{array}{lllllll}
t_{11}^{r} e_{1} & \cdots & t_{1 n}^{r} e_{1} & \cdots & t_{n 1}^{r} e_{n} & \cdots & t_{n n}^{r} e_{n}
\end{array}\right] \\
& E_{r}^{T} \triangleq\left[\begin{array}{lllllll}
t_{11}^{r} e_{1} & \cdots & t_{1 n}^{r} e_{n} & \cdots & t_{n 1}^{r} e_{1} & \cdots & t_{n n}^{r} e_{n}
\end{array}\right] \\
& \vartheta^{r}(z(t)) \triangleq \operatorname{diag}\left\{\vartheta_{11}^{r}\left(z_{1}(t)\right), \cdots, \vartheta_{1 n}^{r}\left(z_{1}(t)\right), \cdots,\right. \\
& \left.\vartheta_{n 1}^{r}\left(z_{n}(t)\right), \cdots, \vartheta_{n n}^{r}\left(z_{n}(t)\right)\right\}, \\
& t_{i j}^{r} \triangleq\left(\left|\bar{r}_{i j}-\underline{r}_{i j}\right| / 2\right)^{\frac{1}{2}},
\end{aligned}
$$

$r \in\{d, a, b, c\}$, and $e_{i} \in \mathbb{R}^{n}$ is a column vector with 1 being its the $i$ th entry and 0 being others. Apparently, $\vartheta^{r}(z(t))$ satisfies $\vartheta^{r T}(z(t)) \vartheta^{r}(z(t)) \leq I$.

Remark 1: In case of computer-based calculations, experiments and simulations, continuous-time networks are often required to be discretized in the implementation process, and this merits the necessity of studying the DMNNs. Note that available results on dynamics analysis problems of DMNNs have been really scattered. Additionally, owing to the statedependent feature of the memristive CWs, it is mathematically difficult to analyze the dynamics of MNNs, and conventional methods cannot be directly employed to investigate the stability and estimation issues for DMNNs. Thanks to the novel SFs (3), we are able to convert the MNN (1) into an equivalent one with parametric uncertainties, on which some traditional robust analysis approaches could be ideally applied.

\section{B. The WTOD Protocol}

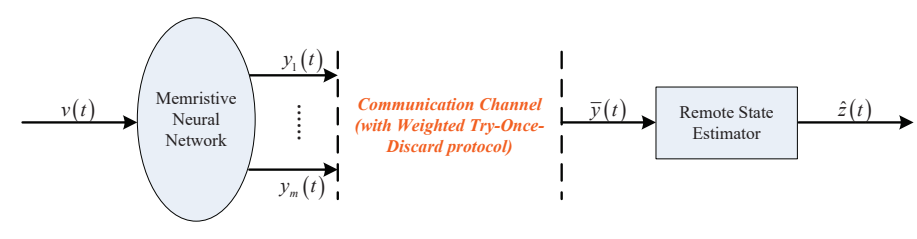

Fig. 1. SEP for an MNN under the WTOD protocol.

As shown in Fig. 1, the measurements $y(t)$ (as the output of the MNN) are sent to the remote estimator via constrained transmission channels. To prevent the data from collisions and maximize the efficiency of data utilization, the WTODP is applied to schedule the data transmission between the MNN and estimator. Under the WTODP, each entry of $y(t)$ has the priority that is directly proportional to the norm of the error between the current measurement and the last storing measurement. At each time instant, only the entry with the highest priority is allowed to be updated. If there are more than one entry being assigned with the highest priority, we can choose an arbitrary one for updating.

Define

$$
\bar{y}(t) \triangleq\left[\begin{array}{llll}
\bar{y}_{1}(t) & \bar{y}_{2}(t) & \ldots & \bar{y}_{m}(t)
\end{array}\right]^{T}
$$

as the received measurement output by the estimator, where $\bar{y}_{s}(t)(s=1,2, \ldots, m)$ is the $s$ th entry of $\bar{y}(t)$. Let $\sigma_{s}$ be the known weight of the $s$ th component, and $\hbar(t) \in\{1,2, \ldots, m\}$ 
be the entry activated at time $t$. Then, $\hbar(t)$ can be determined by

$$
\hbar(t)=\arg \max _{1 \leq s \leq m}(y(t)-\bar{y}(t-1))^{T} \bar{Q}_{s}(y(t)-\bar{y}(t-1))
$$

where $\bar{Q}_{s} \triangleq Q \Psi_{s}, Q \triangleq \operatorname{diag}\left\{\sigma_{1}, \sigma_{2}, \ldots, \sigma_{m}\right\}$ and $\Psi_{s} \triangleq$ $\operatorname{diag}\{\delta(s-1), \delta(s-2), \ldots, \delta(s-m)\}$.

Denote

$$
\begin{aligned}
& \tilde{z}(t) \triangleq\left[\begin{array}{ll}
z^{T}(t) & \bar{y}^{T}(t-1)
\end{array}\right]^{T}, \\
& \tilde{f}(\tilde{z}(t)) \triangleq\left[\begin{array}{ll}
f^{T}(z(t)) & 0
\end{array}\right]^{T}, \\
& \tilde{g}\left(\tilde{z}\left(t-\tau_{1}\right)\right) \triangleq\left[\begin{array}{ll}
g^{T}\left(z\left(t-\tau_{1}\right)\right) & 0
\end{array}\right]^{T}, \\
& \tilde{h}(\tilde{z}(t-\iota)) \triangleq\left[\begin{array}{ll}
h^{T}(z(t-\iota)) & 0
\end{array}\right]^{t}, \\
& \phi_{2}(\varsigma) \triangleq\left[\begin{array}{ll}
\phi_{0}^{T}(\varsigma) & \phi_{1}^{T}
\end{array}\right] \\
& \tilde{v}(t) \triangleq\left[\begin{array}{ll}
v^{T}(t) & v^{T}(t)
\end{array}\right]^{T} .
\end{aligned}
$$

According to the WTODP and the zero order-holders strategy, the actually received measurement can be expressed by

$$
\bar{y}(t)=\Psi_{\hbar(t)} y(t)+\left(I_{m}-\Psi_{\hbar(t)}\right) \bar{y}(t-1),
$$

and $\bar{y}(t)=\phi_{1}$ for $t<0$ where $\phi_{1}$ is known. On the basis of (5), the DMNN in (1) is rewritten as:

$$
\left\{\begin{aligned}
\tilde{z}(t+1)= & \mathcal{D}(t) \tilde{z}(t)+\mathcal{A}(t) \tilde{f}(\tilde{z}(t))+\mathcal{B}(t) \tilde{g}\left(\tilde{z}\left(t-\tau_{1}\right)\right) \\
& +\mathcal{C}(t) \sum_{\iota=1}^{\tau_{2}} \mu_{\iota} \tilde{h}(\tilde{z}(t-\iota))+\mathcal{L}_{1} \tilde{v}(t) \\
\bar{y}(t)= & \mathcal{M} \tilde{z}(t)+\mathcal{L}_{2} v(t) \\
\tilde{z}(\varsigma)= & \phi_{2}(\varsigma), \quad \varsigma=-\tau,-\tau+1, \ldots,-1,0
\end{aligned}\right.
$$

where

$$
\begin{aligned}
& \mathcal{D}(t) \triangleq\left[\begin{array}{cc}
D(z(t)) & 0 \\
\Psi_{\hbar(t)} M & I_{m}-\Psi_{\hbar(t)}
\end{array}\right], \\
& \mathcal{A}(t) \triangleq \operatorname{diag}\{A(z(t)), 0\}, \\
& \mathcal{B}(t) \triangleq \operatorname{diag}\{B(z(t)), 0\}, \\
& \mathcal{C}(t) \triangleq \operatorname{diag}\{C(z(t)), 0\}, \\
& \mathcal{L}_{1} \triangleq \operatorname{diag}\left\{L_{1}, \mathcal{L}_{2}\right\}, \\
& \mathcal{L}_{2} \triangleq \Psi_{\hbar(t)} L_{2}, \\
& \mathcal{M} \triangleq\left[\Psi_{\hbar(t)} M \quad I_{m}-\Psi_{\hbar(t)}\right] .
\end{aligned}
$$

Based on (7), the following RSME is constructed:

$$
\left\{\begin{aligned}
\hat{\tilde{z}}(t+1)= & \overline{\mathcal{D}} \hat{\tilde{z}}(t)+\overline{\mathcal{A}} \tilde{f}(\hat{\tilde{z}}(t)) \\
& +\overline{\mathcal{B}} \tilde{g}\left(\hat{\tilde{z}}\left(t-\tau_{1}\right)\right)+\overline{\mathcal{C}} \sum_{\iota=1}^{\tau_{2}} \mu_{\iota} \tilde{h}(\hat{\tilde{z}}(t-\iota)) \\
& +(\mathcal{K}(t)+\Delta \mathcal{K}(t))(\bar{y}(t)-\mathcal{M} \hat{\tilde{z}}(t)), \\
\hat{\tilde{z}}(\varsigma)= & 0, \quad \varsigma=-\tau,-\tau+1, \ldots,-1,0
\end{aligned}\right.
$$

where

$$
\begin{aligned}
& \hat{\tilde{z}}(t) \triangleq\left[\begin{array}{c}
\hat{z}(t) \\
\hat{\bar{y}}(t-1)
\end{array}\right], \\
& \overline{\mathcal{D}} \triangleq\left[\begin{array}{cc}
D_{0} & 0 \\
\Psi_{\hbar(t)} M & I_{m}-\Psi_{\hbar(t)}
\end{array}\right], \\
& \overline{\mathcal{A}} \triangleq \operatorname{diag}\left\{A_{0}, 0\right\},
\end{aligned}
$$

$$
\begin{aligned}
& \overline{\mathcal{B}} \triangleq \operatorname{diag}\left\{B_{0}, 0\right\}, \\
& \overline{\mathcal{C}} \triangleq \operatorname{diag}\left\{C_{0}, 0\right\}, \\
& \mathcal{K}(t) \triangleq\left[\begin{array}{ll}
K_{z}^{T}(t) & K_{y}^{T}(t)
\end{array}\right]^{T}, \\
& \Delta \mathcal{K}(t) \triangleq\left[\begin{array}{ll}
\Delta K_{z}^{T}(t) & \Delta K_{y}^{T}(t)
\end{array}\right]^{T} .
\end{aligned}
$$

Here, $\hat{\tilde{z}}(t) \in \mathbb{R}^{n+m}$ is the estimate of $\tilde{z}(t), K_{z}(t) \in \mathbb{R}^{n \times m}$ and $K_{y}(t) \in \mathbb{R}^{m \times m}$ are parameters to be determined. $\Delta K_{z}(t)$ and $\Delta K_{y}(t)$ are parameter variations satisfying

$$
\Delta K_{z}(t)=H_{z} F_{z}(t) T_{z}, \Delta K_{y}(t)=H_{y} F_{y}(t) T_{y}
$$

where $H_{z}, H_{y}, T_{z}$ and $T_{y}$ are known matrices, and the unknown matrices $F_{z}$ and $F_{y}$ satisfy $F_{z}^{T}(t) F_{z}(t) \leq I$ and $F_{y}^{T}(t) F_{y}(t) \leq I$.

Let

$$
\begin{aligned}
& e(t) \triangleq \tilde{z}(t)-\hat{\tilde{z}}(t), \\
& \xi(t) \triangleq\left[\begin{array}{ll}
\tilde{v}^{T}(t) \quad v^{T}(t)
\end{array}\right]^{T}, \\
& F(e(t)) \triangleq \tilde{f}(\tilde{z}(t))-\tilde{f}(\hat{\tilde{z}}(t)), \\
& G\left(e\left(t-\tau_{1}\right)\right) \triangleq \tilde{g}\left(\tilde{z}\left(t-\tau_{1}\right)\right)-\tilde{g}\left(\hat{\tilde{z}}\left(t-\tau_{1}\right)\right) \\
& H(e(t-\iota)) \triangleq \tilde{h}(\tilde{z}(t-\iota))-\tilde{h}(\hat{\tilde{z}}(t-\iota)) .
\end{aligned}
$$

Then, the estimation error system with respect to (7) and (8) is

$$
\begin{aligned}
& e(t+1) \\
= & (\overline{\mathcal{D}}-\tilde{\mathcal{K}}(t) \mathcal{M}) e(t)+\Delta \mathcal{D}(t) \tilde{z}(t)+\overline{\mathcal{A}} F(e(t)) \\
& +\Delta \mathcal{A}(t) \tilde{f}(\tilde{z}(t))+\overline{\mathcal{B}} G\left(e\left(t-\tau_{1}\right)\right) \\
& +\Delta \mathcal{B}(t) \tilde{g}\left(\tilde{z}\left(t-\tau_{1}\right)\right)+\sum_{\iota=1}^{\tau_{2}} \mu_{\iota} \overline{\mathcal{C}} H(e(t-\iota)) \\
& +\sum_{\iota=1}^{\tau_{2}} \mu_{\iota} \Delta \mathcal{C}(t) \tilde{h}(\tilde{z}(t-\iota))+\mathcal{L} \xi(t)
\end{aligned}
$$

where

$$
\begin{aligned}
& \Delta \mathcal{D}(t) \triangleq\left[\begin{array}{cc}
\Delta D(z(t)) & 0 \\
0 & 0
\end{array}\right], \\
& \Delta \mathcal{A}(t) \triangleq\left[\begin{array}{cc}
\Delta A(z(t)) & 0 \\
0 & 0
\end{array}\right], \\
& \Delta \mathcal{B}(t) \triangleq\left[\begin{array}{cc}
\Delta B(z(t)) & 0 \\
0 & 0
\end{array}\right], \\
& \Delta \mathcal{C}(t) \triangleq\left[\begin{array}{cc}
\Delta C(z(t)) & 0 \\
0 & 0
\end{array}\right], \\
& \mathcal{L} \triangleq\left[\mathcal{L}_{1}-\tilde{\mathcal{K}}(t) \mathcal{L}_{2}\right], \\
& \tilde{\mathcal{K}}(t) \triangleq \mathcal{K}(t)+\Delta \mathcal{K}(t) .
\end{aligned}
$$

In what follows, we define vectors

$$
\begin{aligned}
& \gamma(t) \triangleq\left[\begin{array}{ll}
\tilde{z}^{T}(t) & e^{T}(t)
\end{array}\right]^{T}, \\
& \tilde{\xi}(t) \triangleq\left[\begin{array}{ll}
\tilde{v}^{T}(t) & \xi^{T}(t)
\end{array}\right]^{T}, \\
& \tilde{F}(\gamma(t)) \triangleq\left[\begin{array}{ll}
\tilde{f}^{T}(\tilde{z}(t)) & F^{T}(e(t))
\end{array}\right]^{T}, \\
& \tilde{G}\left(\gamma\left(t-\tau_{1}\right)\right) \triangleq\left[\begin{array}{ll}
\tilde{g}^{T}\left(\tilde{z}\left(t-\tau_{1}\right)\right) & G^{T}\left(e\left(t-\tau_{1}\right)\right)
\end{array}\right]^{T}, \\
& \tilde{H}(\gamma(t-\iota)) \triangleq\left[\begin{array}{ll}
\tilde{h}^{T}(\tilde{z}(t-\iota)) & H^{T}(e(t-\iota))
\end{array}\right]^{T},
\end{aligned}
$$

and then have the augmented system

$$
\gamma(t+1)=\tilde{\mathcal{D}}(t) \gamma(t)+\tilde{\mathcal{A}}(t) \tilde{F}(\gamma(t))+\tilde{B}(t) \tilde{G}\left(\gamma\left(t-\tau_{1}\right)\right)
$$




$$
+\tilde{\mathcal{C}}(t) \sum_{\iota=1}^{\tau_{2}} \mu_{\iota} \tilde{H}(\gamma(t-\iota))+\tilde{\mathcal{L}} \tilde{\xi}(t)
$$

where

$$
\begin{aligned}
& \tilde{\mathcal{D}}(t) \triangleq \overline{\mathcal{D}}_{\gamma}+\Delta \mathcal{D}_{\gamma}(t), \\
& \tilde{\mathcal{A}}(t) \triangleq \overline{\mathcal{A}}_{\gamma}+\Delta \mathcal{A}_{\gamma}(t), \\
& \tilde{\mathcal{B}}(t) \triangleq \overline{\mathcal{B}}_{\gamma}+\Delta \mathcal{B}_{\gamma}(t), \\
& \tilde{\mathcal{C}}(t) \triangleq \overline{\mathcal{C}}_{\gamma}+\Delta \mathcal{C}_{\gamma}(t), \\
& \tilde{\mathcal{L}} \triangleq \overline{\mathcal{L}}_{\gamma}+\Delta \mathcal{L}_{\gamma}, \\
& \overline{\mathcal{D}}_{\gamma} \triangleq \operatorname{diag}\{\overline{\mathcal{D}}, \overline{\mathcal{D}}-\mathcal{K}(t) \mathcal{M}\}, \\
& \overline{\mathcal{A}}_{\gamma} \triangleq \operatorname{diag}\{\overline{\mathcal{A}}, \overline{\mathcal{A}}\}, \\
& \overline{\mathcal{B}}{ }_{\gamma} \triangleq \operatorname{diag}\{\overline{\mathcal{B}}, \overline{\mathcal{B}}\}, \\
& \overline{\mathcal{C}}_{\gamma} \triangleq \operatorname{diag}\{\overline{\mathcal{C}}, \overline{\mathcal{C}}\}, \\
& \overline{\mathcal{L}} \triangleq\left[\mathcal{L}_{1} \quad-\mathcal{K}(t) \mathcal{L}_{2}\right], \\
& \overline{\mathcal{L}}_{\gamma} \triangleq \operatorname{diag}\left\{\mathcal{L}_{1}, \overline{\mathcal{L}}\right\}, \\
& \Delta \mathcal{L}_{\gamma} \triangleq \operatorname{diag}\{0, \Delta \overline{\mathcal{L}}\}, \\
& \Delta \overline{\mathcal{L}} \triangleq\left[\begin{array}{ll}
0 & -\Delta \mathcal{K}(t) \mathcal{L}_{2}
\end{array}\right], \\
& \Delta \mathcal{D}_{\gamma}(t) \triangleq\left[\begin{array}{ll}
\Delta \mathcal{D}(t) & 0 \\
\Delta \mathcal{D}(t) & -\Delta \mathcal{K}(t) \mathcal{M}
\end{array}\right], \\
& \Delta \mathcal{C}_{\gamma}(t) \triangleq\left[\begin{array}{ll}
\Delta \mathcal{C}(t) & 0 \\
\Delta \mathcal{C}(t) & 0
\end{array}\right], \\
& \Delta \mathcal{A}_{\gamma}(t) \triangleq\left[\begin{array}{ll}
\Delta \mathcal{A}(t) & 0 \\
\Delta \mathcal{A}(t) & 0
\end{array}\right], \\
& \Delta \mathcal{B}_{\gamma}(t) \triangleq\left[\begin{array}{ll}
\Delta \mathcal{B}(t) & 0 \\
\Delta \mathcal{B}(t) & 0
\end{array}\right] .
\end{aligned}
$$

For presentation convenience, we now introduce the following assumptions and definitions.

Assumption $1: \phi_{2}(\varsigma)(\varsigma=-\tau,-\tau+1, \cdots,-1,0)$ satisfy

$$
\phi_{2}^{T}(\varsigma) P^{-1}(\varsigma) \phi_{2}(\varsigma) \leq 1
$$

where matrices $P(\varsigma)(\varsigma=-\tau,-\tau+1, \cdots,-1,0)$ are known and positive-definite.

Assumption 2: The external stochastic disturbance $v(t)$ satisfies the following condition

$$
v^{T}(t) \Gamma_{v}^{-1}(t) v(t) \leq 1
$$

where matrix $\Gamma_{v}(t)$ is known and positive-definite.

Assumption 3: The neuron AFs $f(\cdot), g(\cdot)$ and $h(\cdot)$ satisfy $f(0)=g(0)=h(0)=0$ and following Lipschitz conditions:

$$
\begin{aligned}
\|f(s)-f(t)\| & \leq\left\|\Gamma_{1}(s-t)\right\|, \\
\|g(s)-g(t)\| & \leq\left\|\Gamma_{2}(s-t)\right\|, \\
\|h(s)-h(t)\| & \leq\left\|\Gamma_{3}(s-t)\right\|
\end{aligned}
$$

for all $s, t \in \mathbb{R}^{n}$, where $\Gamma_{1}, \Gamma_{2}$ and $\Gamma_{3}$ are known constant matrices.

Definition 1: Let the matrix (ellipsoid matrices) sequence $P(t) \in \mathbb{R}^{(n+m) \times(n+m)}\left(t \in \mathbb{N}^{+}\right)$be given. System (10) is said to meet the $P(t)$-dependent constraint if

$$
\mathcal{R}(t) \triangleq \gamma^{T}(t) P^{-1}(t) \gamma(t) \leq 1
$$

holds for $t \in \mathbb{N}^{+}$.
In this paper, we are set to design a RSME that is capable of confining the estimates of the DMNN (1) to a CER under the UNBBNs. Such an aim is accomplished in two steps. First, for the given matrix sequence $\{P(t)\}_{t \in \mathbb{N}^{+}}$, we like to find the sufficient condition that ensures that the RSME exists and, subsequently, (10) meets the $P(t)$-dependent constraint (14). Second, we like to minimize the trace of $P(t)$ through appropriately selecting $\mathcal{K}(t)$ that satisfies the aforementioned sufficient condition.

\section{MAin Results}

This section aims at establishing sufficient conditions that guarantee that the $P(t)$-dependent constraint (14) is satisfied by system (10). Then, a recursive algorithm is proposed to determine $\mathcal{K}(t)$ under the WTODP. To start with, we present the following useful lemmas to benefit the subsequent derivation.

Lemma 1: [2] Let $\varphi_{0}(\cdot), \varphi_{1}(\cdot), \cdots, \varphi_{p}(\cdot)$ be quadratic functions of $\mathbf{s} \in \mathbb{R}^{n}: \varphi_{j}(\mathbf{s}) \triangleq \mathbf{s}^{T} Z_{i} \mathbf{s}(i=0,1, \cdots, p)$ and $Z_{j}=Z_{j}^{T}$. If there exist $\varrho_{1} \geq 0, \varrho_{2} \geq 0, \cdots, \varrho_{p} \geq 0$ such that $Z_{0}-\sum_{i=1}^{p} \varrho_{i} Z_{i} \leq 0$, then

$$
\varphi_{1}(\mathbf{s}) \leq 0, \varphi_{2}(\mathbf{s}) \leq 0, \cdots, \varphi_{p}(\mathbf{s}) \leq 0 \rightarrow \varphi_{0}(\mathbf{s}) \leq 0 .
$$

Lemma 2: [2] Given matrices $\Omega_{1}, \Omega_{2}, \Omega_{3}, \Omega_{1}=\Omega_{1}^{T}$ and $\Omega_{2}>0$, then

$$
\Omega_{1}+\Omega_{3}^{T} \Omega_{2}^{-1} \Omega_{3}<0
$$

iff

$$
\left[\begin{array}{cc}
\Omega_{1} & \Omega_{3}^{T} \\
\Omega_{3} & -\Omega_{2}
\end{array}\right]<0 .
$$

Lemma 3: [36] Given matrices $\mathcal{N}=\mathcal{N}^{T}, \mathcal{H}, \mathcal{E}$ and $\mathcal{F}^{T} \mathcal{F} \leq I$, then

$$
\mathcal{N}+\mathcal{H} \mathcal{F} \mathcal{E}+(\mathcal{H} \mathcal{F} \mathcal{E})^{T}<0
$$

holds iff there exists scalar $\mu>0$ such that

$$
\mathcal{N}+\mu \mathcal{H} \mathcal{H}^{T}+\mu^{-1} \mathcal{E}^{T} \mathcal{E}<0
$$

or

$$
\left[\begin{array}{ccc}
\mathcal{N} & \mu \mathcal{H} & \mathcal{E}^{T} \\
* & -\mu I & 0 \\
* & * & -\mu I
\end{array}\right]<0 .
$$

For the convenience of presentation, we denote $\tilde{\mathcal{H}}_{r} \triangleq\left[\begin{array}{cc}\mathcal{H}_{r}^{T} & 0\end{array}\right]^{T}, \quad \tilde{E}_{r} \triangleq\left[\begin{array}{ll}E_{r} & 0\end{array}\right], \quad P(t) \triangleq L(t) L^{T}(t)$.

The following theorem gives a sufficient condition for the solvability of the concerned SEP.

Theorem 1: Consider the DMNN (1) with given estimator (8 and matrices sequences $\{P(t)>0\}_{t \in \mathbb{N}^{+}}$. If there exist $\mathcal{K}(t), \varepsilon(t), \lambda_{i}(t) \quad(i \in\{1,2,3,4\})$ and $\epsilon_{s}(t)(s \in$ $\left.\{1,2, \cdots, m\}, t \in \mathbb{N}^{+}\right)$satisfying

$$
\left[\begin{array}{ccc}
\tilde{\Omega}(t) & * & * \\
\varepsilon(t) \tilde{\mathcal{H}}^{T} & -\varepsilon(t) I & * \\
\tilde{\mathcal{E}} & 0 & -\varepsilon(t) I
\end{array}\right]<0
$$

where

$$
\tilde{\Omega}(t) \triangleq\left[\begin{array}{cc}
-\Lambda(t) & * \\
\bar{\Phi}(t) & -P(t+1)
\end{array}\right],
$$




$$
\begin{aligned}
& \bar{\Phi}(t) \triangleq\left[\begin{array}{llllllll}
0 & \overline{\mathcal{D}}_{\gamma} L(t) & 0 & 0 & \overline{\mathcal{L}}_{\gamma} & \overline{\mathcal{A}}_{\gamma} & \overline{\mathcal{B}}_{\gamma} & \overline{\mathcal{C}}_{\gamma}
\end{array}\right], \\
& \Lambda(t) \triangleq \operatorname{diag}\left\{1-\lambda_{1}(t)-\lambda_{2}(t)-5 \lambda_{3}(t)\right. \text {, } \\
& \lambda_{1}(t) I-\lambda_{4}(t) L^{T}(t) \tilde{\Gamma}_{1}^{T} \tilde{\Gamma}_{1} L(t), \\
& \lambda_{2}(t) I-\lambda_{4}(t) L^{T}\left(t-\tau_{1}\right) \tilde{\Gamma}_{2}^{T} \tilde{\Gamma}_{2} L\left(t-\tau_{1}\right), \\
& \left.\lambda_{4}(t) \Omega_{0}(t), \lambda_{3}(t) \tilde{\Gamma}_{v}^{-1}(t), \lambda_{4}(t) I, \lambda_{4}(t) I, \lambda_{4}(t) I\right\} \\
& +\mathcal{N}^{T}(t) \sum_{s=1}^{m} \epsilon_{s}(t)\left(Q\left(\Psi_{s}-\Psi_{\hbar(t)}\right)\right) \mathcal{N}(t), \\
& \tilde{\mathcal{H}} \triangleq\left[\begin{array}{ccccccccc}
0 & 0 & 0 & 0 & 0 & 0 & 0 & 0 & \tilde{\mathcal{H}}_{\mathcal{D}}^{T} P^{T}(t) \\
0 & 0 & 0 & 0 & 0 & 0 & 0 & 0 & \tilde{\mathcal{H}}_{\mathcal{A}}^{T} P^{T}(t) \\
0 & 0 & 0 & 0 & 0 & 0 & 0 & 0 & \tilde{\mathcal{H}}_{\mathcal{B}}^{T} P^{T}(t) \\
0 & 0 & 0 & 0 & 0 & 0 & 0 & 0 & \tilde{\mathcal{H}}_{\mathcal{C}}^{T} P^{T}(t) \\
0 & 0 & 0 & 0 & 0 & 0 & 0 & 0 & \tilde{\mathcal{H}}_{\mathcal{L}}^{T} P^{T}(t)
\end{array}\right]^{T}, \\
& \tilde{\mathcal{E}} \triangleq\left[\begin{array}{ccccccccc}
0 & \tilde{E}_{\mathcal{D}} L(t) & 0 & 0 & 0 & 0 & 0 & 0 & 0 \\
0 & 0 & 0 & 0 & 0 & \tilde{E}_{\mathcal{A}} & 0 & 0 & 0 \\
0 & 0 & 0 & 0 & 0 & 0 & \tilde{E}_{\mathcal{B}} & 0 & 0 \\
0 & 0 & 0 & 0 & 0 & 0 & 0 & \tilde{E}_{\mathcal{C}} & 0 \\
0 & 0 & 0 & 0 & T_{\mathcal{L}} & 0 & 0 & 0 & 0
\end{array}\right], \\
& \mathcal{R}(0)=\gamma^{T}(0) P^{-1}(0) \gamma(0)=\phi_{2}^{T}(0) P^{-1}(0) \phi_{2}(0) \leq 1 . \\
& \bar{\gamma}_{\tau_{2}} \triangleq\left[\begin{array}{llll}
\gamma^{T}(t-1) & \gamma^{T}(t-2) & \cdots & \gamma^{T}\left(t-\tau_{2}\right)
\end{array}\right]^{T}
\end{aligned}
$$

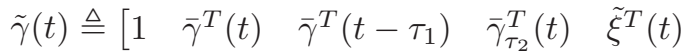

$$
\begin{aligned}
& \tilde{F}^{T}(\gamma(t)) \quad \tilde{G}^{T}\left(\gamma\left(t-\tau_{1}\right)\right) \\
& \left.\sum_{\iota=1}^{\tau_{2}} \mu_{\iota} \tilde{H}^{T}(\gamma(t-\iota))\right]^{T},
\end{aligned}
$$$$
\tilde{\mathcal{H}}_{\mathcal{D}} \triangleq\left[\begin{array}{cc}
\tilde{\mathcal{H}}_{d} & 0 \\
\tilde{\mathcal{H}}_{d} & -\mathcal{H}_{\mathcal{K}}
\end{array}\right] \text {, }
$$$$
\tilde{\mathcal{H}}_{\mathcal{A}} \triangleq\left[\begin{array}{ll}
\tilde{\mathcal{H}}_{a} & 0 \\
\tilde{\mathcal{H}}_{a} & 0
\end{array}\right],
$$$$
\tilde{\mathcal{H}}_{\mathcal{B}} \triangleq\left[\begin{array}{ll}
\tilde{\mathcal{H}}_{b} & 0 \\
\tilde{\mathcal{H}}_{b} & 0
\end{array}\right],
$$$$
\tilde{\mathcal{H}}_{\mathcal{C}} \triangleq\left[\begin{array}{ll}
\tilde{\mathcal{H}}_{c} & 0 \\
\tilde{\mathcal{H}}_{c} & 0
\end{array}\right] \text {, }
$$$$
\tilde{\mathcal{H}}_{\mathcal{L}} \triangleq\left[\begin{array}{c}
0 \\
-\mathcal{H}_{\mathcal{K}}
\end{array}\right] \text {, }
$$$$
\tilde{E}_{\mathcal{D}} \triangleq \operatorname{diag}\left\{\tilde{E}_{d}, T_{\mathcal{K}} \mathcal{M}\right\}, \quad \tilde{E}_{\mathcal{A}} \triangleq \operatorname{diag}\left\{\tilde{E}_{a}, 0\right\},
$$$$
\tilde{E}_{\mathcal{B}} \triangleq \operatorname{diag}\left\{\tilde{E}_{b}, 0\right\} \text {, }
$$$$
\tilde{E}_{\mathcal{C}} \triangleq \operatorname{diag}\left\{\tilde{E}_{c}, 0\right\} \text {, }
$$$$
T_{\mathcal{K}} \triangleq\left[\begin{array}{ll}
T_{x}^{T} & T_{y}^{T}
\end{array}\right]^{T},
$$$$
T_{\mathcal{L}} \triangleq\left[\begin{array}{lll}
0 & 0 & T_{\mathcal{K}} \mathcal{L}_{2}
\end{array}\right],
$$$$
\mathcal{H}_{\mathcal{K}} \triangleq \operatorname{diag}\left\{H_{x}, H_{y}\right\} \text {, }
$$$$
\overline{\mathcal{N}} \triangleq\left[\begin{array}{lll}
M & -I & 0
\end{array}\right],
$$$$
\check{\mathcal{L}} \triangleq\left[\begin{array}{lllll}
L_{2} & 0 & 0 & 0 & 0
\end{array}\right],
$$$$
\bar{\mu} \triangleq \sum_{\iota=1}^{\tau_{2}} \mu_{\iota},
$$$$
\mathcal{N}(t) \triangleq\left[\begin{array}{llllllll}
0 & \overline{\mathcal{N}} L(t) & 0 & 0 & \check{\mathcal{L}} & 0 & 0 & 0
\end{array}\right],
$$$$
\Omega_{0}(t) \triangleq \bar{\mu} \operatorname{diag}\left\{\mu_{1} \tilde{\Gamma}_{3}^{T} \tilde{\Gamma}_{3}, \mu_{2} \tilde{\Gamma}_{3}^{T} \tilde{\Gamma}_{3}, \cdots, \mu_{\tau_{2}} \tilde{\Gamma}_{3}^{T} \tilde{\Gamma}_{3}\right\},
$$$$
\tilde{\Gamma}_{1} \triangleq \operatorname{diag}\left\{\Gamma_{1}, \Gamma_{1}, \Gamma_{1}, \Gamma_{1}\right\} \text {, }
$$$$
\tilde{\Gamma}_{2} \triangleq \operatorname{diag}\left\{\Gamma_{2}, \Gamma_{2}, \Gamma_{2}, \Gamma_{2}\right\} \text {, }
$$$$
\tilde{\Gamma}_{3} \triangleq \operatorname{diag}\left\{\Gamma_{3}, \Gamma_{3}, \Gamma_{3}, \Gamma_{3}\right\} \text {, }
$$$$
\tilde{\Gamma}_{v}(t) \triangleq I_{5} \otimes \Gamma_{v}(t) \text {, }
$$

then system (10) satisfies $P(t)$-dependent constraints with $\mathcal{K}(t)\left(t \in \mathbb{N}^{+}\right)$. we have

$$
\gamma(t+1)=\Phi(t) \tilde{\gamma}(t)
$$

and thus

$$
\begin{aligned}
& \gamma^{T}(t+1) P^{-1}(t+1) \gamma(t+1)-1 \\
= & \tilde{\gamma}^{T}(t)\left(\Phi^{T}(t) P^{-1}(t+1) \Phi(t)\right. \\
& -\operatorname{diag}\{1,0,0,0,0,0,0,0\}) \tilde{\gamma}(t)
\end{aligned}
$$

where

$$
\Phi(t) \triangleq\left[\begin{array}{llllllll}
0 & \tilde{\mathcal{D}}(t) L(t) & 0 & 0 & \tilde{\mathcal{L}} & \tilde{\mathcal{A}}(t) & \tilde{\mathcal{B}}(t) & \tilde{\mathcal{C}}(t)
\end{array}\right]
$$

It follows from Assumption 2 and the WTODP (5) that

$$
\left\{\begin{array}{l}
\|\bar{\gamma}(t)\|^{2} \leq 1 \\
\left\|\bar{\gamma}\left(t-\tau_{1}\right)\right\|^{2} \leq 1 \\
\tilde{\xi}^{T}(t) \tilde{\Gamma}_{v}^{-1}(t) \tilde{\xi}(t) \leq 5, \\
(y(t)-\bar{y}(t-1))^{T} Q\left(\Psi_{s}-\Psi_{\hbar(t)}\right) \\
\times(y(t)-\bar{y}(t-1)) \leq 0, \quad s=1,2, \ldots, m
\end{array}\right.
$$

which, by (17)-(21), is rearranged as follows (in terms of $\tilde{\gamma}(t)$ ):

$$
\left\{\begin{array}{l}
\tilde{\gamma}^{T}(t) \operatorname{diag}\{-1, I, 0,0,0,0,0,0\} \tilde{\gamma}(t) \leq 0, \\
\tilde{\gamma}^{T}(t) \operatorname{diag}\{-1,0, I, 0,0,0,0,0\} \tilde{\gamma}(t) \leq 0 \\
\tilde{\gamma}^{T}(t) \operatorname{diag}\left\{-5,0,0,0, \tilde{\Gamma}_{v}^{-1}(t), 0,0,0\right\} \tilde{\gamma}(t) \leq 0 \\
\tilde{\gamma}^{T}(t) \mathcal{N}^{T}(t) Q\left(\Psi_{s}-\Psi_{\hbar(t)}\right) \\
\times \mathcal{N}(t) \tilde{\gamma}(t) \leq 0, \quad s=1,2, \ldots, m
\end{array}\right.
$$

Noticing Assumption 3, we have

$$
\begin{aligned}
& \tilde{F}^{T}(\gamma(t)) \tilde{F}(\gamma(t)) \leq \gamma^{T}(t) \Gamma_{1}^{T} \Gamma_{1} \gamma(t), \\
& \begin{aligned}
\tilde{G}^{T}\left(\gamma\left(t-\tau_{1}\right)\right) \tilde{G}\left(\gamma\left(t-\tau_{1}\right)\right) \\
\quad \leq \gamma^{T}\left(t-\tau_{1}\right) \Gamma_{2}^{T} \Gamma_{2} \gamma\left(t-\tau_{1}\right),
\end{aligned}
\end{aligned}
$$


and

$$
\begin{aligned}
& \left(\sum_{\iota=1}^{\tau_{2}} \mu_{\iota} \tilde{H}(\gamma(t-\iota))\right)^{T}\left(\sum_{\iota=1}^{\tau_{2}} \mu_{\iota} \tilde{H}(\gamma(t-\iota))\right) \\
\leq & \left(\sum_{\iota=1}^{\tau_{2}} \mu_{\iota}\right) \sum_{\iota=1}^{\tau_{2}} \mu_{\iota} \tilde{H}^{T}(\gamma(t-\iota)) \tilde{H}(\gamma(t-\iota)) \\
\leq & \bar{\mu} \sum_{\iota=1}^{\tau_{2}} \mu_{\iota} \gamma^{T}(t-\iota) \Gamma_{3}^{T} \Gamma_{3} \gamma(t-\iota) \\
= & \bar{\mu} \bar{\gamma}_{\tau_{2}}^{T}(t) \Omega_{0}(t) \bar{\gamma}_{\tau_{2}}(t) .
\end{aligned}
$$

Furthermore, (23)-(25) are expressed as

$$
\begin{aligned}
& \tilde{F}^{T}(\gamma(t)) \tilde{F}(\gamma(t)) \leq \tilde{\gamma}^{T}(t) \Omega_{1}(t) \tilde{\gamma}(t) \leq 0 \\
& \tilde{G}^{T}\left(\gamma\left(t-\tau_{1}\right)\right) \tilde{G}\left(\gamma\left(t-\tau_{1}\right)\right) \leq \tilde{\gamma}^{T}(t) \Omega_{2}(t) \tilde{\gamma}(t) \leq 0 \\
& \left(\sum_{\iota=1}^{\tau_{2}} \mu_{\iota} \tilde{H}(\gamma(t-\iota))\right)^{T}\left(\sum_{\iota=1}^{\tau_{2}} \mu_{\iota} \tilde{H}(\gamma(t-\iota))\right) \\
& \leq \tilde{\gamma}^{T}(t) \Omega_{3}(t) \tilde{\gamma}(t) \leq 0
\end{aligned}
$$

where

$$
\begin{aligned}
& \Omega_{1}(t) \triangleq \operatorname{diag}\left\{0,-L^{T}(t) \Gamma_{1}^{T} \Gamma_{1} L(t), 0,0,0, I, 0,0\right\}, \\
& \Omega_{2}(t) \triangleq \operatorname{diag}\left\{0,0,-L^{T}\left(t-\tau_{1}\right) \Gamma_{2}^{T} \Gamma_{2} L\left(t-\tau_{1}\right), 0,0,0, I, 0\right\}, \\
& \Omega_{3}(t) \triangleq \operatorname{diag}\left\{0,0,0, \Omega_{0}(t), 0,0,0, I\right\} .
\end{aligned}
$$

with $\Omega_{0}(t)$ being defined in (15). Then,

$$
\tilde{\gamma}^{T}(t) \Omega(t) \tilde{\gamma}(t) \leq 0
$$

where

$$
\begin{aligned}
\Omega(t) \triangleq \operatorname{diag}\left\{0,-L^{T}(t) \Gamma_{1}^{T} \Gamma_{1} L(t),\right. & \\
& \left.-L^{T}\left(t-\tau_{1}\right) \Gamma_{2}^{T} \Gamma_{2} L\left(t-\tau_{1}\right), \Omega_{0}(t), 0, I, I, I\right\} .
\end{aligned}
$$

Using Lemma 1, if there exist positive scalars $\lambda_{1}(t), \lambda_{2}(t)$, $\lambda_{3}(t), \lambda_{4}(t)$ and $\epsilon_{s}(t)(s=1,2, \cdots, m)$ such that

$$
\begin{aligned}
& \Phi^{T}(t) P^{-1}(t+1) \Phi(t) \\
& -\lambda_{1}(t) \operatorname{diag}\{-1, I, 0,0,0,0,0,0\} \\
& -\lambda_{2}(t) \operatorname{diag}\{-1,0, I, 0,0,0,0,0\} \\
& -\lambda_{3}(t) \operatorname{diag}\left\{-5,0,0,0, \tilde{\Gamma}_{v}^{-1}(t), 0,0,0\right\} \\
& -\lambda_{4}(t) \Omega(t)-\operatorname{diag}\{1,0,0,0,0,0,0,0\} \\
& -\mathcal{N}^{T}(t) \sum_{s=1}^{m} \epsilon_{s}(t)\left(Q\left(\Psi_{s}-\Psi_{\hbar(t)}\right)\right) \mathcal{N}(t) \leq 0,
\end{aligned}
$$

then inequality $\gamma^{T}(t+1) P^{-1}(t+1) \gamma(t+1)-1 \leq 0$ holds by referring to inequalities (22) and (29).

Let us convert (30) into the compact form below:

$$
\Phi^{T}(t) P^{-1}(t+1) \Phi(t)-\Lambda(t) \leq 0 .
$$

Then, in view of (31) and Lemma 2, we obtain

$$
\left[\begin{array}{cc}
-\Lambda(t) & * \\
\Phi(t) & -P(t+1)
\end{array}\right] \leq 0 .
$$

Furthermore, to eliminate uncertainties embedded in (32), we rewrite (32) as

$$
\left[\begin{array}{cc}
-\Lambda(t) & * \\
\bar{\Phi}(t) & -P(t+1)
\end{array}\right]+\tilde{\mathcal{H}} \tilde{\mathcal{F}} \tilde{\mathcal{E}}+(\tilde{\mathcal{H}} \tilde{\mathcal{F}} \tilde{\mathcal{E}})^{T} \leq 0
$$

where

$$
\begin{aligned}
\tilde{\mathcal{F}} \triangleq\left[\begin{array}{ccccc}
\mathcal{F}_{\mathcal{D}} & 0 & 0 & 0 & 0 \\
0 & \vartheta^{a}(z(t)) & 0 & 0 & 0 \\
0 & 0 & \vartheta^{b}(z(t)) & 0 & 0 \\
0 & 0 & 0 & \vartheta^{c}(z(t)) & 0 \\
0 & 0 & 0 & 0 & \mathcal{F}_{\mathcal{K}}
\end{array}\right], \\
\mathcal{F}_{\mathcal{D}} \triangleq \operatorname{diag}\left\{\vartheta^{d}(z(t)), \mathcal{F}_{\mathcal{K}}\right\} \\
\mathcal{F}_{\mathcal{K}} \triangleq \operatorname{diag}\left\{F_{z}, F_{y}\right\} .
\end{aligned}
$$

By resorting to Lemma 3, we observe that (15) is ensured by (33). Thus, it can be obtained from (15) that $\mathcal{R}(t+1) \leq 1$. This completes the induction. Hence, (14) is guaranteed with parameter $\mathcal{K}(t)$.

Theorem 1 guarantees the existence of a CER that embraces all possible $z(t+1)$, where the state estimate obtained from (8) serves as the center of ellipsoid.

In the following corollary, by exerting the convex optimization approach, we will present an iterative algorithm to optimize this ellipsoid and compute the optimal state estimator gain.

Corollary 1: The constraint matrix $P(t)$ on system (10) is minimized (in the sense of the matrix trace) if there exist $\mathcal{K}(t)$, $\varepsilon(t), \lambda_{i}(t)(i \in\{1,2,3,4\})$ and $\epsilon_{s}(t)(s \in\{1,2, \cdots, m\}, t \in$ $\left.\mathbb{N}^{+}\right)$such that the following optimization problem

$$
\min _{(P(t+1), \mathcal{K}(t), \ell(t))} \operatorname{tr}\{P(t+1)\}
$$

is solvable s.t. (15), where

$$
\begin{aligned}
\ell(t) \triangleq\{\varepsilon(t)\} & \cup\left\{\lambda_{1}(t), \lambda_{2}(t), \lambda_{3}(t), \lambda_{4}(t)\right\} \\
& \cup\left\{\epsilon_{1}(t), \epsilon_{2}(t), \cdots, \epsilon_{m}(t)\right\} .
\end{aligned}
$$

Proof: The proof is easily accessible from Theorem 1 and is therefore omitted for brevity.

It should be mentioned that inequalities (15), are linear to variables $P(t), \mathcal{K}(t), \varepsilon(t), \lambda_{i}(t)(i \in\{1,2,3,4\})$ and $\epsilon_{s}(t)$ $(s \in\{1,2, \cdots, m\})$. Actually, it is seen from Corollary 1 that the aforementioned optimization problem can be well addressed via standard semi-definite programming techniques. Therefore, by means of Corollary 1, we can summarize the computational algorithm for the state estimation gains $\{\mathcal{K}(t)>0\}_{t \in \mathbb{N}^{+}}$in Algorithm 1 as follows.

\footnotetext{
Algorithm 1 Computational Algorithm for $\{\mathcal{K}(t)>0\}_{t \in \mathbb{N}^{+}}$

Step 1. Initialization: Set $t=0$ and given $N$ and $P(\varsigma)$.

Step 2. Calculate $L(\varsigma)$ based on $P(\varsigma)=L(\varsigma) L^{T}(\varsigma)$ for $\varsigma=t-\tau_{1}$ and $t-\tau_{2} \leq \varsigma \leq t$.

Step 3. Solve (34) s. t. (15). Then, $\mathcal{K}(t)$ and $P(t+1)$ are obtained based on the solution of (34).

Step 4. Set $t=t+1$. If $t>N$, exit. Otherwise, go to Step 2 .
}

Remark 2: In the paper, the set-membership SEP for DMNNs has been effectively coped with under HTDs and the WTODP. One can observe from Theorem 1 and Algorithm 1 that, in the pursuit of the RSME, all significant factors (including the state-dependent parameters, HTDs, noise information, estimation accuracy, WTODP) are fully reflected in the above analysis, and a comprehensive framework is formulated, under 
which the desired estimator gains are derived by taking into account factors that sophisticate the concerned model.

Remark 3: The state estimation problem for artificial neural networks has received a large amount of research attention and a large body of results has been available in the literature. In comparison with the existing literature, the main results of this paper exhibits the following distinctive features: 1) the set-membership state estimation problem discussed in this paper is new in the sense that the random gain variation and the WTODP are taken into careful consideration; 2) a novel yet unified estimation scheme is developed to tackle the mathematical complexities stemming from the state-dependent switching behaviors and hybrid time-delays; and 3) the design algorithm of the desired filters is recursive and can be realized online.

\section{An Illustrative EXAmple}

This section provides a demonstrative example to validate the proposed estimation approach.

Consider (1) with parameters:

$$
\begin{aligned}
& d_{1}\left(z_{1}(\cdot)\right)= \begin{cases}0.990, & \left|z_{1}(\cdot)\right|>0.3, \\
0.930, & \left|z_{1}(\cdot)\right| \leq 0.3,\end{cases} \\
& d_{2}\left(z_{2}(\cdot)\right)= \begin{cases}0.420, & \left|z_{2}(\cdot)\right|>0.3, \\
0.860, & \left|z_{2}(\cdot)\right| \leq 0.3,\end{cases} \\
& a_{11}\left(z_{1}(\cdot)\right)= \begin{cases}0.060, & \left|z_{1}(\cdot)\right|>0.3, \\
0.050, & \left|z_{1}(\cdot)\right| \leq 0.3,\end{cases} \\
& a_{12}\left(z_{1}(\cdot)\right)= \begin{cases}-0.330, & \left|z_{1}(\cdot)\right|>0.3, \\
-0.350, & \left|z_{1}(\cdot)\right| \leq 0.3,\end{cases} \\
& a_{21}\left(z_{2}(\cdot)\right)= \begin{cases}-0.230, & \left|z_{2}(\cdot)\right|>0.3, \\
-0.200, & \left|z_{2}(\cdot)\right| \leq 0.3,\end{cases} \\
& a_{22}\left(z_{2}(\cdot)\right)= \begin{cases}0.060, & \left|z_{2}(\cdot)\right|>0.3, \\
0.040, & \left|z_{2}(\cdot)\right| \leq 0.3,\end{cases} \\
& b_{11}\left(z_{1}(\cdot)\right)=\left\{\begin{aligned}
-0.300, & \left|z_{1}(\cdot)\right|>0.3, \\
0.240, & \left|z_{1}(\cdot)\right| \leq 0.3,
\end{aligned}\right. \\
& b_{12}\left(z_{1}(\cdot)\right)= \begin{cases}0.005, & \left|z_{1}(\cdot)\right|>0.3, \\
0.015, & \left|z_{1}(\cdot)\right| \leq 0.3,\end{cases} \\
& b_{21}\left(z_{2}(\cdot)\right)=\left\{\begin{aligned}
-0.310, & \left|z_{2}(\cdot)\right|>0.3, \\
0.270, & \left|z_{2}(\cdot)\right| \leq 0.3,
\end{aligned}\right. \\
& b_{22}\left(z_{2}(\cdot)\right)= \begin{cases}0.020, & \left|z_{2}(\cdot)\right|>0.3, \\
0.060, & \left|z_{2}(\cdot)\right| \leq 0.3,\end{cases} \\
& c_{11}\left(z_{1}(\cdot)\right)= \begin{cases}0.015, & \left|z_{1}(\cdot)\right|>0.3, \\
0.045, & \left|z_{1}(\cdot)\right| \leq 0.3,\end{cases} \\
& c_{12}\left(z_{1}(\cdot)\right)= \begin{cases}0.050, & \left|z_{1}(\cdot)\right|>0.3, \\
0.010, & \left|z_{1}(\cdot)\right| \leq 0.3,\end{cases} \\
& c_{21}\left(z_{2}(\cdot)\right)=\left\{\begin{aligned}
0.260, & \left|z_{2}(\cdot)\right|>0.3, \\
-0.300, & \left|z_{2}(\cdot)\right| \leq 0.3,
\end{aligned}\right.
\end{aligned}
$$

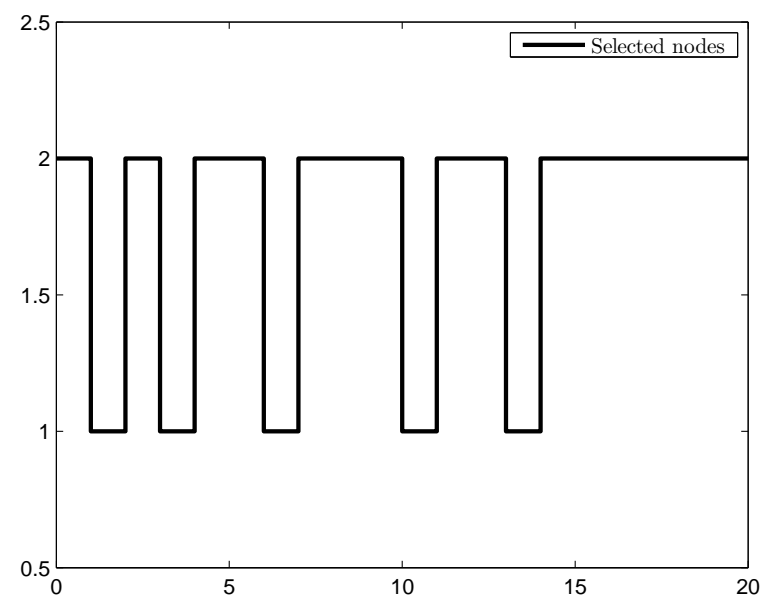

Fig. 2. The selected nodes of MNNs

$$
\begin{aligned}
c_{22}\left(z_{2}(\cdot)\right) & =\left\{\begin{array}{rr}
0.015, & \left|z_{2}(\cdot)\right|>0.3, \\
0.035, & \left|z_{2}(\cdot)\right| \leq 0.3,
\end{array}\right. \\
\Gamma_{1} & =\Gamma_{2}=\Gamma_{3}=\left[\begin{array}{cc}
0.25 & 0 \\
0 & 0.36
\end{array}\right], \\
\tau_{1} & =1, \tau_{2}=3 .
\end{aligned}
$$

The weight values in the WTODP are $\sigma_{1}=1.0$ and $\sigma_{2}=0.8$. The bounded noise is set as $v(t)=\sqrt{0.02} \cos (t)$, and thus we have $\Gamma_{v}(t)=0.02 I$ for $t \in \mathbb{N}^{+}$. Furthermore, $\phi_{2}(\varsigma)=\left[\begin{array}{llll}3 & 3 & 1 & 1\end{array}\right]^{T}, P(\varsigma)=\operatorname{diag}\{9,9,1,1,9,9,1,1\}$ $(\varsigma=-\tau,-\tau+1, \ldots, 0)$.

Using Matlab YALMIP 3.0 Toolbox, the optimization problem (34) is solved subject to (15), and the corresponding demonstration results are given in Figs. 2-6. Fig. 2 reveals the impact from the neural states on the selected neural nodes, while Fig. 3 and Fig. 4 plot the actual and estimated trajectories of $z_{1}(t), z_{2}(t)$ and their estimation errors, respectively. The actual and estimated values of $\bar{y}_{1}(t), \bar{y}_{2}(t)$ and their estimation errors are respectively displayed in Fig. 5 and Fig. 6. The obtained results demonstrates that our proposed estimation scheme is indeed effective.

\section{CONCLuSions}

The set-membership SEP has been solved in this paper for DMNNs with HTDS under WTODP. The WTODP has been utilized to mitigate the unnecessary network congestion occurred in the channel between the DMNNs and estimator. To cater for possible execution errors, estimator gains are contaminated by bounded parameter fluctuations. A RSME has then been devised to achieve the estimate of the DMNN susceptible to the UBBNs and WTOD. By means of the RMIs, sufficient conditions have been pinned down to ensure the existence of the desired RSME. Then, an optimization problem has been formulated by minimizing the CER (with respect to estimation errors) under the WTODP. Finally, simulation results illustrate the feasibility of our RSME. Further research topics would 


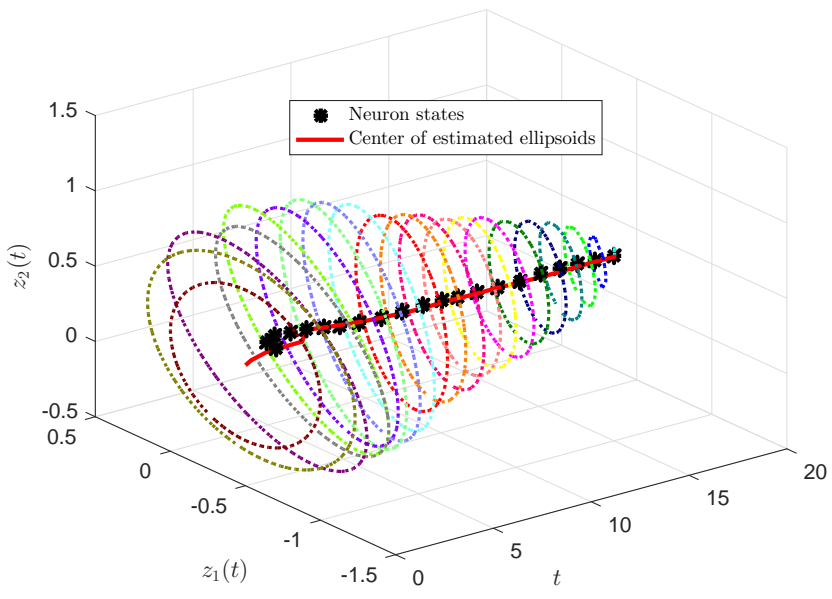

Fig. 3. The actual and estimated trajectories of $z_{1}(t)$ and $z_{2}(t)$

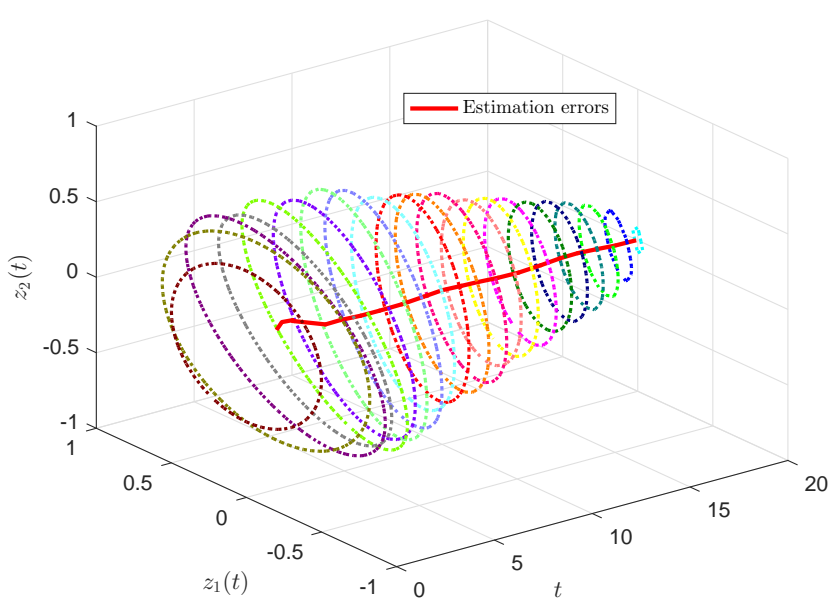

Fig. 4. The estimation errors of $z_{1}(t)$ and $z_{2}(t)$

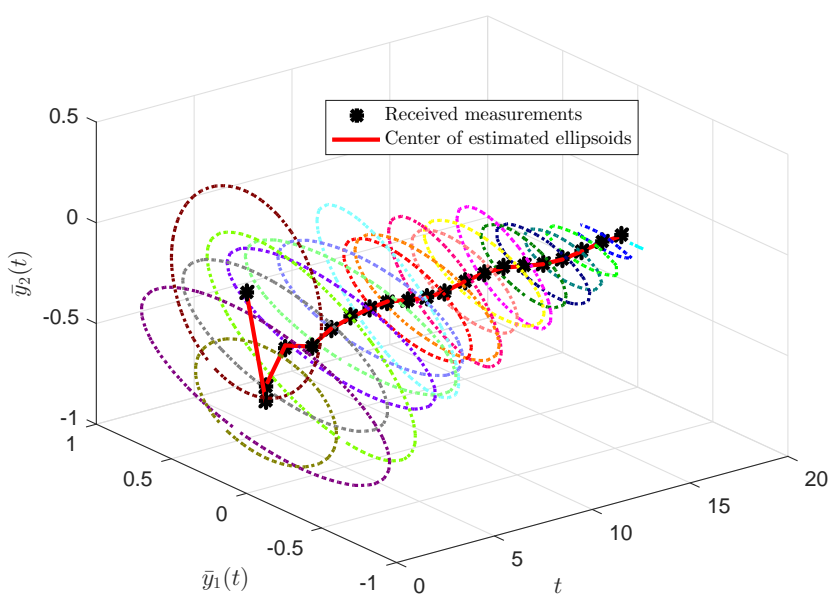

Fig. 5. The actual and estimated values of $\bar{y}_{1}(t)$ and $\bar{y}_{2}(t)$

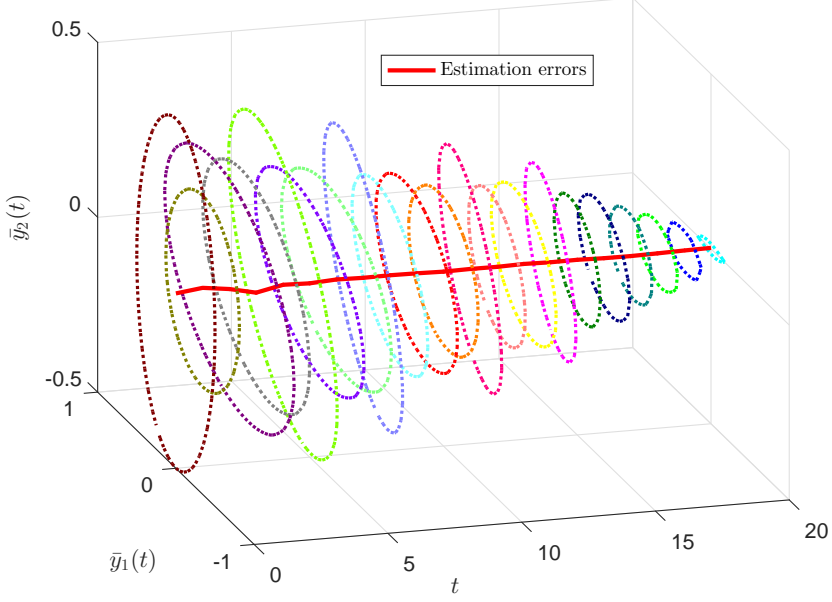

Fig. 6. The estimation errors of $\bar{y}_{1}(t)$ and $\bar{y}_{2}(t)$

include the extension of the main results to 1) the partialnodes-based set-membership SEP of complex networks under communication protocols [22], [23], [48]; 2) the recursive SEP of DMNN with network-enhanced complexities [9], [10]; and 3 ) the improvement of the state estimation performance by using some latest optimization algorithms [24], [25].

\section{REFERENCES}

[1] S. Arik, A modified Lyapunov functional with application to stability of neutral-type neural networks with time delays, Journal of the Franklin Institute, vol. 356, no. 1, pp. 276-291, 2019.

[2] S. P. Boyd, L. E. Ghaoui, E. Feron, and V. Balakrishnan, Linear Matrix Inequalities in System and Control Theory. Philadelphia, PA: SIAM (Studies in Applied Mathematics), 1994.

[3] W. Chen, D. Ding, J. Mao, H. Liu and N. Hou, Dynamical performance analysis of communication-embedded neural networks: A survey, $\mathrm{Neu}$ rocomputing, vol. 346, no. 21, pp. 3-11, Jun. 2019.

[4] L. Cheng, W. Liu, C. Yang, T. Huang, Z.-G. Hou and M. Tan, A neuralnetwork-based controller for piezoelectric-actuated stick-slip devices, IEEE Transactions on Industrial Electronics, vol. 65, no. 3, pp. 25982607, Mar. 2018

[5] M. C. F. Donkers, W. P. M. H. Heemels, N. van de Wouw, and L. Hetel, Stability analysis of networked control systems using a switched linear systems approach, IEEE Transactions on Automatic Control, vol. 56, no. 9, pp. 2101-2115, 2011.

[6] E. Fridman, New Lyapunov-Krasovskii functionals for stability of linear retarded and neutral type systems, Systems Control and Letters, vol. 43, no. 4, pp. 309-319, 2001.

[7] L. El Ghaoui and G. Calafiore, Robust filtering for discrete-time systems with bounded noise and parametric uncertainty, IEEE Transactions on Automatic Control, vol. 46, no. 7, pp. 1302-1313, 2001.

[8] M. Hernandez-Gonzalez, M. V. Basin and E. A. Hernandez-Vargas, Discrete-time high-order neural network identifier trained with highorder sliding mode observer and unscented Kalman filter, Neurocomputing, in press, DOI: 10.1016/j.neucom.2019.12.005.

[9] J. Hu, Z. Wang, G.-P. Liu and H. Zhang, Variance-constrained recursive state estimation for time-varying complex networks with quantized measurements and uncertain inner coupling, IEEE Transactions on Neural Networks and Learning Systems, vol. 31, no. 6, pp. 1955-1967, 2020.

[10] J. Hu, Z. Wang, G.-P. Liu, C. Jia and J. Williams, Event-triggered recursive state estimation for dynamical networks under randomly switching topologies and multiple missing measurements, Automatica, vol. 115, art. no. $108908,2020$.

[11] X. Hu, G. Feng, S. Duan, and L. Liu, A memristive multilayer cellular neural network with applications to image processing, IEEE Transations on Neural Networks and Learning Systems, vol. 28, no. 8, pp. 18891901, 2017. 
[12] H. R. Karimi and H. Gao, New delay-dependent exponential $H_{\infty}$ synchronization for uncertain neural networks with mixed time delays, IEEE Transactions on Systems, Man, and Cybernetics, Part B: Cybernetics, vol. 40, no. 1, pp. 173-185, Jul. 2009.

[13] L. H. Keel and S. P. Bhattacharyya, Robust, fragile, or optimal?, IEEE Transactions on Automatic Control, vol. 42, no. 8, pp. 1098-1105, 1997.

[14] O. M. Kwon, M. J. Park, S. M. Lee, J. H. Park, and E. J. Cha, Stability for neural networks with time-varying delays via some new approaches, IEEE Transactions on Neural Networks and Learning Systems, vol. 24, no. 2, pp. 181-193, 2013.

[15] L. Li, X. Shi and J. Liang, Synchronization of impulsive coupled complex-valued neural networks with delay: The matrix measure method, Neural Networks, vol. 117, pp. 285-294, Sept. 2019.

[16] J. Li, Z. Wang, H. Dong and F. Han, Delay-distribution-dependent state estimation for neural networks under stochastic communication protocol with uncertain transition probabilities, Neural Networks, vol. 130, pp. 143-151, 2020.

[17] X. Li, F. Han, N. Hou, H. Dong and H. Liu, Set-membership filtering for piecewise linear systems with censored measurements under RoundRobin protocol, International Journal of Systems Science, in press, DOI: 10.1080/00207721.2020.1768453.

[18] H. Liu, Z. Wang, B. Shen and H. Dong, Delay-distribution-dependent $H_{\infty}$ state estimation for discrete-time memristive neural networks with mixed time-delays and fading measurements, IEEE Transactions on Cybernetics, vol. 50, no. 2, pp. 440-451, 2020.

[19] H. Liu, Z. Wang, W. Fei and J. Li, $H_{\infty}$ and $l_{2}-l_{\infty}$ state estimation for discrete-time delayed memristive neural networks on finite horizon: The Round-Robin protocol, Neural Networks, vol. 132, pp. 121-130, 2020.

[20] S. Liu, Z. Wang, G. Wei and M. Li, Distributed set-membership filtering for multi-rate systems under the Round-Robin scheduling over sensor networks, IEEE Transactions on Cybernetics, vol. 50, no. 5, pp. 1910 1920, 2020.

[21] Y. Liu, B. Shen and H. Shu, Finite-time resilient $H_{\infty}$ state estimation for discrete-time delayed neural networks under dynamic event-triggered mechanism, Neural Networks, vol. 121, pp. 356-365, Jan. 2020.

[22] Y. Liu, Z. Wang, L. Ma and F. E. Alsaadi, A partial-nodes-based information fusion approach to state estimation for discrete-time delayed stochastic complex networks, Information Fusion, vol. 49, pp. 240-248, Sept. 2019.

[23] Y. Liu, Z. Wang, Y. Yuan and W. Liu, Event-triggered partial-nodesbased state estimation for delayed complex networks with bounded distributed delays, IEEE Transactions on Systems, Man, and Cybernetics: Systems, vol. 49, no. 6, pp. 1088-1098, Jun. 2019.

[24] Y. Liu, Q. Cheng, Y. Gan, Y. Wang, Z. Li and J. Zhao, Multi-objective optimization of energy consumption in crude oil pipeline transportation system operation based on exergy loss analysis, Neurocomputing, vol. 332, pp. 100-110, Mar. 2019.

[25] Y. Liu, S. Chen, B. Guan and P. Xu, Layout optimization of largescale oil-gas gathering system based on combined optimization strategy, Neurocomputing, vol. 332, pp. 159-183, Mar. 2019.

[26] Y. V. Pershin and M. Di Ventra, Memory effects in complex materials and nanoscale systems, Advances in Physics, vol. 60, no. 2, pp. 145-227, 2011.

[27] R. Sakthivel, P. Vadivel, K. Mathiyalagan, A. Arunkumar and M. Sivachitra, Design of state estimator for bidirectional associative memory neural networks with leakage delays, Information Sciences, vol. 296, pp. 263-274, 2015.

[28] B. Shen, Z. Wang, D. Wang and H. Liu, Distributed state-saturated recursive filtering over sensor networks under Round-Robin protocol, IEEE Transactions on Cybernetics, vol. 50, no. 8, pp. 3605-3615, Aug. 2020.

[29] H. Shen, S. Huo, J. Cao and T. Huang, Generalized state estimation for Markovian coupled networks under Round-Robin protocol and redundant channels, IEEE Transactions on Cybernetics, vol. 49, no. 4 , pp. 1292-1301, 2019.

[30] Y. Shen, Z. Wang, B. Shen, F. E. Alsaadi and F. E. Alsaadi, Fusion estimation for multi-rate linear repetitive processes under weighted TryOnce-Discard protocol, Information Fusion, vol. 55, pp. 281-291, 2020.

[31] K. L. Shepard and V. Narayanan, Noise in deep submicron digital design, Proceeding IEEE/ACM International Conference ComputerAided Design, pp. 524-531, 1996.

[32] L. Tian, Y. Cheng, C. Yin, D. Ding, Y. Song and L. Bai, Design of the MOI method based on the artificial neural network for crack detection, Neurocomputing, vol. 226, pp. 80-89, 2017.

[33] S. N. Truong, K. V. Pham, W. Yang, and K. S. Min, Sequential memristor crossbar for neuromorphic pattern recognition, IEEE Transactions on Nanotechnology, vol. 15, no. 6, pp. 922-930, 2016.
[34] V. Ugrinovskii and E. Fridman, A Round-Robin type protocol for distributed estimation with $H_{\infty}$ consensus, Systems and Control Letters, vol. 69, pp. 103-110, 2014.

[35] X. Wan, L. Xu, H. Fang, and G. Ling, Robust non-fragile $H_{\infty}$ state estimation for discrete-time genetic regulatory networks with Markov jump delays and uncertain transition probabilities, Neurocomputing, vol. 154, pp. 162-173, 2015.

[36] Y. Wang, L. Xie, and C. E. de Souza, Robust control of a class of uncertain nonlinear systems, Systems and Control Letters, vol. 19, no. 2, pp. 139-149, 1992.

[37] S. Wen, Z. Zeng, and T. Huang, Exponential stability analysis of memristor-based recurrent neural networks with time-varying delays, Neurocomputing, vol. 97, pp. 233-240, 2012.

[38] Z. Wu, Z. Xu, P. Shi, M. Chen, and H. Su, Non-fragile state estimation of quantized complex networks with switching topologies, IEEE Transations on Neural Networks and Learning Systems, vol. 29, no. 10, pp. 5111-5121, 2018.

[39] W. Xu, J. Cao, M. Xiao, D. W. C. Ho and G. Wen, A new framework for analysis on stability and bifurcation in a class of neural networks with discrete and distributed delays, IEEE Transactions on Cybernetics, vol. 45, no. 10, pp. 2224-2236, Oct. 2015.

[40] Y. Xu, C. Liu, J.-Y. Li, C.-Y. Su and T. Huang, Finite-Horizon $H_{\infty}$ state estimation for time-varying neural networks with periodic inner coupling and measurements scheduling, IEEE Transactions on Systems Man Cybernetics-Systems, vol. 50, no. 1, pp. 211-219, Jan. 2020.

[41] G.-H. Yang and W. Che, Non-fragile $H_{\infty}$ filter design for linear continuous-time systems, Automatica, vol. 44, no. 11, pp. 2849-2859, 2008.

[42] G.-H. Yang and J. Wang, Robust non-fragile Kalman filtering for uncertain linear systems with estimator gain uncertainty, IEEE Transactions on Automatic Control, vol. 46, no. 2, pp. 343-348, 2001.

[43] L. Zhang, Y. Zhu and W. X. Zheng, Energy-to-peak state estimation for Markov jump RNNs with time-varying delays via nonsynchronous filter with nonstationary mode transitions, IEEE Transactions on Neural Networks and Learning Systems, vol. 26, no. 10, pp. 2346-2356, 2015.

[44] X.-M. Zhang, Q.-L. Han and X. Ge, An overview of neuronal state estimation of neural networks with time-varying delays, Information Sciences, vol. 478, pp. 83-99, 2019.

[45] W. Zhang, C. Li, T. Huang, and X. He, Synchronization of memristorbased coupling recurrent neural networks with time-varying delays and impulses, IEEE Transations on Neural Networks and Learning Systems, vol. 26, no. 12, pp. 3308-3313, 2015.

[46] D. Zhang, W. Cai, L. Xie, and Q. Wang, Non-fragile distributed filtering for T-S fuzzy systems in sensor networks, IEEE Transations on Fuzzy Systems, vol. 23, no. 5, pp. 1883-1890, 2015.

[47] Z.-M. Zhang, Y. He, M. Wu and Q.-G. Wang, Exponential synchronization of neural networks with time-varying delays via dynamic intermittent output feedback control, IEEE Transactions on Systems Man Cybernetics-Systems, vol. 49, no. 3, pp. 612-622, Mar. 2019.

[48] Z. Zhao, Z. Wang, L. Zou and J. Guo, Set-Membership filtering for timevarying complex networks with uniform quantisations over randomly delayed redundant channels, International Journal of Systems Science, in press, DOI: 10.1080/00207721.2020.1814898.

[49] L. Zou, Z. Wang, H. Geng and X. Liu, Set-membership filtering subject to impulsive measurement outliers: A recursive algorithm, IEEE/CAA Journal of Automatica Sinica, vol. 8, no. 2, pp. 377-388, Feb. 2021.

[50] L. Zou, Z. Wang, Q.-L. Han and D. H. Zhou, Moving horizon estimation of networked nonlinear systems with random access protocol, IEEE Transactions on Systems, Man, and Cybernetics: Systems, in press, DOI: 10.1109/TSMC.2019.2918002.

[51] L. Zou, Z. Wang, Q.-L. Han and D. H. Zhou, Full information estimation for time-varying systems subject to Round-Robin scheduling: A recursive filter approach, IEEE Transactions on Systems, Man, and Cybernetics: Systems, in press, DOI: 10.1109/TSMC.2019.2907620. 


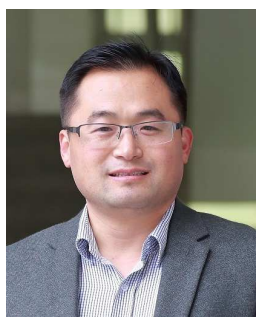

Hongjian Liu received his B.Sc. degree in applied mathematics in 2003 from Anhui University, Hefei, China, and the M.Sc. degree in detection technology and automation equipment in 2009 from Anhui Polytechnic University, Wuhu, China, and the $\mathrm{Ph} . \mathrm{D}$. degree in control science and engineering in 2018 from Donghua University, Shanghai, China. In 2016, he was a Research Assistant with the Department of Mathematics, Texas A\&M University at Qatar, Doha, Qatar, for two months. From March 2017 to March 2018, he was a Visiting Scholar in the Department of Information Systems and Computing, Brunel University London, UK. He is currently a Professor in the School of Mathematics and Physics, Anhui Polytechnic University, Wuhu, China.

Dr. Liu's current research interests include filtering theory, memristive neural networks, and network communication systems. He is a very active reviewer for many international journals.

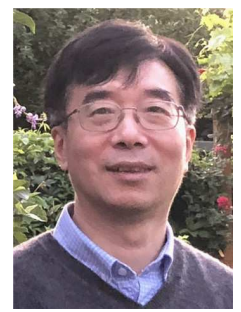

Zidong Wang (SM'03-F'14) was born in Jiangsu, China, in 1966. He received the B.Sc. degree in mathematics in 1986 from Suzhou University, Suzhou, China, and the M.Sc. degree in applied mathematics in 1990 and the Ph.D. degree in electrical engineering in 1994, both from Nanjing University of Science and Technology, Nanjing, China.

$\mathrm{He}$ is currently Professor of Dynamical Systems and Computing in the Department of Computer Science, Brunel University London, U.K. From 1990 to 2002, he held teaching and research appointments in universities in China, Germany and the UK. Prof. Wang's research interests include dynamical systems, signal processing, bioinformatics, control theory and applications. He has published more than 600 papers in international journals. He is a holder of the Alexander von Humboldt Research Fellowship of Germany, the JSPS Research Fellowship of Japan, William Mong Visiting Research Fellowship of Hong Kong.

Prof. Wang serves (or has served) as the Editor-in-Chief for International Journal of Systems Science, the Editor-in-Chief for Neurocomputing, and an Associate Editor for 12 international journals including IEEE Transactions on Automatic Control, IEEE Transactions on Control Systems Technology, IEEE Transactions on Neural Networks, IEEE Transactions on Signal Processing, and IEEE Transactions on Systems, Man, and Cybernetics-Part C. He is a Member of the Academia Europaea, a Fellow of the IEEE, a Fellow of the Royal Statistical Society and a member of program committee for many international conferences.

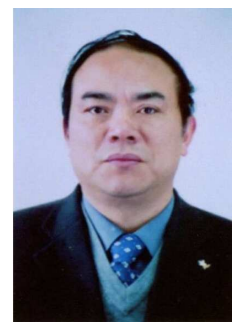

Weiyin Fei is a Professor with the School of Mathematics and Physics, Anhui Polytechnic University, Wuhu, Anhui, China. He received the B.S. degree in 1986 from Anhui Normal University, Wuhu, Anhui, China, the M.S. degree and the Ph.D. degree from Donghua University, Shanghai, China, in 1996 and 2002, respectively. His research interests include stochastic control, financial mathematics, and stochastic differential equation with applications. Dr. Fei is the author of more than 150 research papers.

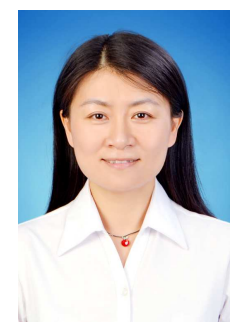

Hongli Dong received the Ph.D. degree in control science and engineering from the Harbin Institute of Technology, Harbin, China, in 2012.

From 2009 to 2010, she was a Research Assistant with the Department of Applied Mathematics, City University of Hong Kong, Hong Kong. From 2010 to 2011, she was a Research Assistant with the Department of Mechanical Engineering, The University of Hong Kong, Hong Kong. From 2011 to 2012, she was a Visiting Scholar with the Department of Information Systems and Computing, Brunel University London, London, U.K. From 2012 to 2014, she was an Alexander von Humboldt Research Fellow with the University of Duisburg-Essen, Duisburg, Germany. She is currently a Professor with the Artificial Intelligence Energy Research Institute, Northeast Petroleum University, Daqing, China. She is also the Director of the Heilongjiang Provincial Key Laboratory of Networking and Intelligent Control, Daqing. Her current research interests include robust control and networked control systems.

Dr. Dong is a very active reviewer for many international journals. 